\title{
Smart shape memory alloy chiral honeycomb.
}

\begin{abstract}
An auxetic (or negative Poisson's ratio) material expands in all directions when pulled in only one, behaving in an opposite way compared with "classical" materials. A structure not superimposable with its mirror image is defined as chiral. A chiral structural honeycomb (noncentresymmetric) features auxeticity, i.e., a negative Poisson's ratio behaviour in the plane. Although chirality is common in nature and organic chemistry, it is an unusual characteristic in structural materials and components. We have manufactured truss assemblies based on cells of chiral honeycomb topology using shape memory alloy (SMA) ribbons as core material. The main objective of this work is to obtain a new functional structure combining the chiral honeycomb topology and shape memory alloys as a new concept of smart cellular solid. The chiral SMA honeycomb can be used in new types of deployable antenna reflectors, allowing the compression of the structure in a small volume of space for subsequent deployment. The new honeycomb concept could also be used in packaging applications to store strain energy during an impact loading and as a core for a sandwich structure for damping or for crashworthiness.
\end{abstract}

Keyword: Auxetic; Chiral; Deployable; Poisson's ratio; Pseudoelasticity. 